\title{
Propiedades psicométricas de la escala de intensificación de demandas del trabajo a partir de una muestra colombiana
}

\author{
Juan Sandoval-Reyes; Fernando Riveros Munévar; Edison Jair Duque Oliva
}

How to cite this article:

Sandoval-Reyes, J., Riveros Munévar, F., Duque Oliva, E.J., (2020). Psychometric Properties of the Intensification of Job Demands Scale based on a sample of Colombian workers. Acta Colombiana de Psicología, 23(2), 40-51. http://www. doi.org/10.14718/ACP.2020.23.2.3

Recibido, mayo 29/2019; Concepto de evaluación, septiembre 24/2019; Aceptado, enero 16/2020

\author{
Juan Sandoval-Reyes* \\ Universidad de La Sabana, Chía, Colombia \\ ORCID: https://orcid.org/0000-0001-6458-6301 \\ Fernando Riveros Munévar \\ Universidad de La Sabana, Chía, Colombia \\ ORCID: https://orcid.org/0000-0002-0414-674X \\ Edison Jair Duque Oliva \\ Universidad Nacional de Colombia, Bogotá, Colombia \\ ESAI Business School, Guayas, Ecuador. \\ ORCID: https://orcid.org/0000-0003-4949-6118
}

\begin{abstract}
Resumen
La aceleración del ritmo de gestión organizacional plantea nuevos desafíos para las empresas, que pueden reflejarse en la intensificación de las demandas del trabajo y en que las personas expresen un aumento tanto en los niveles de intensidad en el desarrollo de sus tareas como en la sensación de una mayor presión del tiempo en su contexto laboral. Teniendo esto en cuenta, el objetivo del presente estudio fue examinar en una muestra de trabajadores colombianos las evidencias de validez de constructo y confiabilidad de la versión en español de la escala Intensification of Job Demands Scale (IDS), desarrollada por Kubicek et al. en 2015 para evaluar cinco demandas del trabajo sujetas a la intensificación - la muestra estuvo compuesta por 420 profesionales del sector de servicios de la salud en Bogotá, Colombia—. Para esto, se establecieron los estadísticos descriptivos, así como los índices de discriminación de los ítems, la estructura interna del instrumento mediante análisis factorial exploratorio y confirmatorio, y las evidencias de validez de constructo. Como resultado, se mantiene la estructura original de cinco factores de la prueba, que explican el $47.12 \%$ de la varianza total; y, por medio de la utilización de modelos de ecuaciones estructurales, se evidencia un adecuado ajuste y parsimonia del modelo en congruencia con la propuesta teórica de la escala. Se concluye que esta versión en español de la IDS posee propiedades psicométricas adecuadas y que puede contribuir a la investigación en la región sobre la intensificación del trabajo como una demanda asociada al deterioro en la salud y al bienestar en los trabajadores.

Palabras clave: condiciones de trabajo, propiedades psicométricas, análisis factorial, ecuaciones de modelos estructurales (SEM).
\end{abstract}

\section{Psychometric Properties of the Intensification of Job Demands Scale based on a sample of Colombian workers}

Abstract

The acceleration of the pace of organizational management poses new challenges that can be expressed as an intensification of job demands, and people express it as an increase in the intensity levels of their task development, and a feeling of

* Facultad de Psicología, Universidad de La Sabana, Campus del Puente del Común, Km. 7, Autopista Norte de Bogotá. Chía, Cundinamarca 250001, Colombia. Tel.: (+0571) 8615555 Ext.28409. juan.sandoval1@unisabana.edu.co 
a greater time pressure in their work context. The objective of this study was to examine, in a sample of Colombian workers, the evidence of construct validity and reliability of the Spanish version of the Intensification of Job Demands Scale (IDS) developed by Kubicek, Paškvan and Korunka in 2015, which evaluates five job demands subject to intensification.

The sample consisted of 420 healthcare professionals located in Bogotá, Colombia. Descriptive statistics, item discrimination indexes, internal structure through exploratory and confirmatory factor analysis, and evidence of construct validity were established. The original five-factor structure of the test was maintained explaining $47.12 \%$ of the total variance. By using Structural Equation Models (SEM), an adequate adjustment and parsimony of the model in congruence with the theoretical proposal of the scale is evidenced. This Spanish version of the IDS has adequate psychometric properties and it can contribute to research in the country, on the intensification of work as a demand that is associated with the impairment of workers' health and well-being.

Keywords: working conditions; psychometric properties; factor analysis; structural equation models (SEM).

\section{Introducción}

Conocer los efectos que producen las características y condiciones del trabajo en las personas es un aspecto de gran interés para los directivos y profesionales responsables de la gestión de personas en las organizaciones, de modo que entender, explicar e incluso predecir los niveles de bienestar, motivación y desempeño de los empleados de una organización ha sido siempre un ideal para las disciplinas de la administración y de la psicología organizacional y del trabajo (Paškvan \& Kubicek, 2017).

Existe suficiente evidencia (Bakker et al., 2003; Bakker et al., 2007; Hakanen et al., 2006) que demuestra cómo las características de una tarea producen un profundo impacto en el bienestar de un trabajador, que puede ser positivo, como el aprendizaje, la motivación y el compromiso hacia la tarea y la organización; o negativo, como el agotamiento, la tensión emocional y los problemas de salud, sobre todo cuando algunas de las características son vistas como demandas o exigencias (Franke, 2015; Krause et al., 2005; Kubicek et al., 2013; Lesener et al., 2019).

Ahora bien, el mundo viene enfrentando distintos cambios socioeconómicos, así como múltiples avances tecnológicos y un progresivo aumento de la competitividad entre las empresas, factores que inevitablemente influyen en los contextos organizacionales y, a su vez, representan un reto tanto para directivos como para trabajadores (Paškvan $\&$ Kubicek, 2017). En la Figura 1 se resumen algunos de los cambios a nivel organizacional e individual que se han presentado como resultado de los cambios descritos a nivel global.

De igual forma, el cambio hacia una economía basada en servicios, la transición de economías nacionales cerradas a economías globales y la flexibilización de las estructuras organizacionales que fomentan nuevas prácticas de gestión son fenómenos representativos de los cambios que el mundo del trabajo ha vivido desde la década de los años setenta del siglo pasado. En los últimos años uno de los indicadores

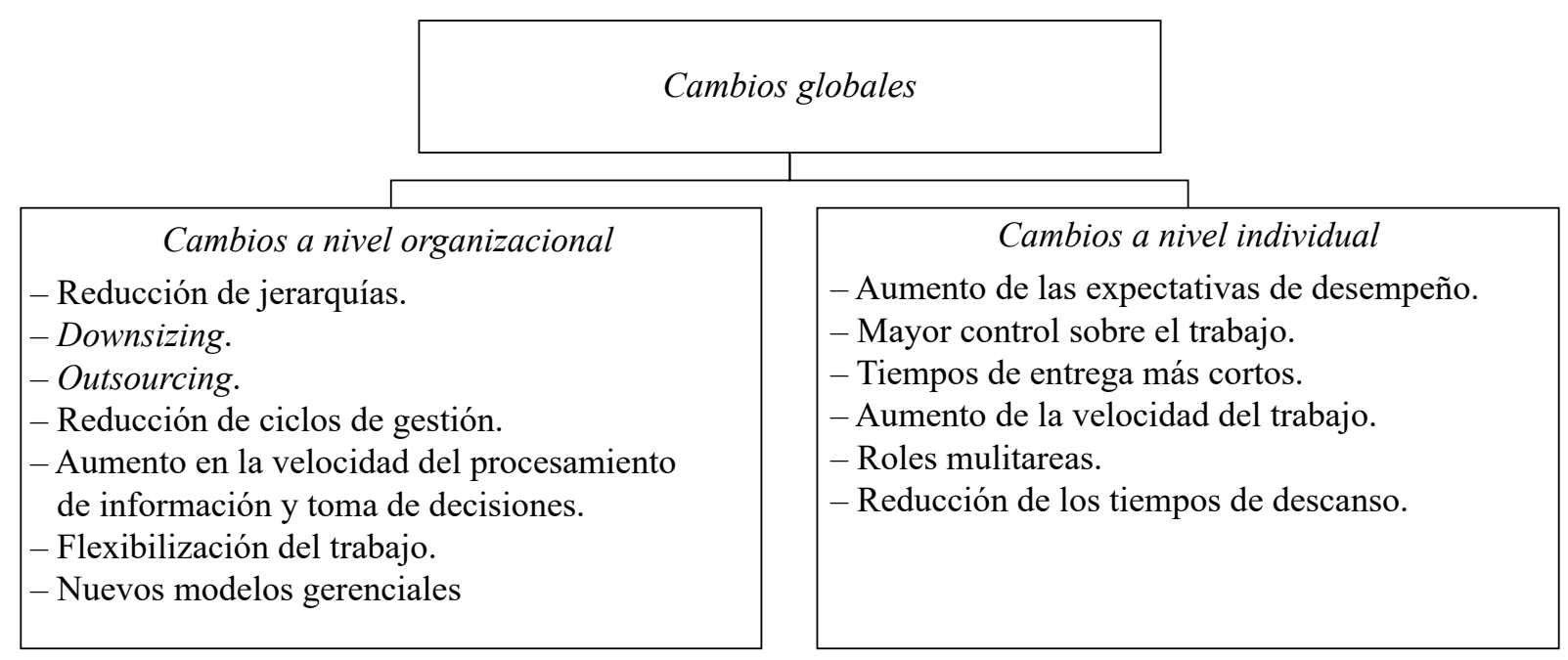

Figura 1. Cambios en el mundo del trabajo. Fuente: tomado y adaptado de Paskvan y Kubicek (2017). 
clave en el proceso de cambio ha sido la aceleración del ritmo con el que este se viene presentando.

Al respecto, autores como Rosa $(2003,2013)$ plantean que la aceleración es una de las consecuencias más importantes del desarrollo del capitalismo, y por tanto proponen que dicha aceleración en el cambio social —un fenómeno que se puede analizar desde una estructura de tres dimensiones: aceleración tecnológica, social y del ritmo de vida - tiene grandes efectos en la vida laboral (Flecker et al., 2017), sobre todo en las condiciones de trabajo de las personas; de modo que los ajustes en los ciclos de gestión organizacional y en las formas del trabajo también significan un impacto en las exigencias para la realización de las tareas de la empresa, aspecto que requiere ser estudiado de manera precisa y rigurosa (Bakker \& Demerouti, 2014).

Teniendo esto en cuenta, a partir del Modelo DemandasRecursos —o Job Demands-Resource Model, de Bakker y Demerouti (2007)—, Franke (2015) propone que los fenómenos relacionados con la aceleración de los ritmos de gestión organizacional pueden plantearse como una intensificación de las demandas del trabajo, lo cual puede entenderse como mayores niveles de intensidad en el desarrollo de las tareas y sentimientos de mayor presión del tiempo en los contextos de trabajo. De hecho, Paškvan y Kubicek (2017) definen la intensificación del trabajo como "un constructo multifacético caracterizado por la necesidad de trabajar más rápido, por enfrentar tiempos de respuesta más cortos, por la reducción de los tiempos de descanso y [por] la necesidad de realizar varias tareas simultáneamente" (p. 26). En este sentido, la búsqueda del incremento en la eficiencia organizacional despliega nuevas formas del trabajo que no solo impulsan la velocidad de los ciclos de gestión, sino que también extienden la jornada de trabajo y aumentan el control sobre el desempeño (Neirotti, 2018).

A partir de esto, la intensificación del trabajo se puede operacionalizar como el aumento de los esfuerzos físicos y cognitivos que el individuo debe emplear para realizar su trabajo (Bathini \& Kandathil, 2019), lo que se refleja en el ritmo de trabajo, en la presión por tomar decisiones más rápidas y en la sensación que el día de trabajo no termina (Granter et al., 2019).

A partir de lo anterior, y como resultado del creciente interés por estudiar el fenómeno, en la literatura científica sobre el tema se ha podido identificar un modelo de medición de las demandas del trabajo sujetas a intensificación, el modelo desarrollado por Kubicek et al. (2015), en el que se detallan cinco dimensiones conceptuales:

a. La intensificación de las demandas de esfuerzo laboral, que hace referencia al incremento en la cantidad de esfuerzo que los empleados necesitan invertir para hacer su trabajo; intensificación mediada por la presión adicional que ponen los directivos sobre los empleados para tratar de obtener una ventaja competitiva, reducir los ciclos de gestión y aumentar la velocidad de los procesos de decisión y producción. Esta intensificación está positivamente relacionada con la presión de tiempo y con las nuevas formas de trabajo que implican que el trabajo nunca se detiene (Kelliher \& Anderson, 2008).

b. La intensificación de las demandas de la planeación y la autonomía en el trabajo, que refiere a las nuevas lógicas organizacionales en las que se reduce el control directo y se incrementan los requerimientos de toma de decisiones y de planeación autónoma. Acá, debido a los rápidos cambios en las prácticas de gestión, las empresas han reaccionado con el aumento de la velocidad para la toma de decisiones y con la implementación de estructuras organizacionales más flexibles, lo cual ha generado una nueva lógica de control sobre las personas en la que se reduce el control directo de los jefes y se aumenta la autonomía en todos los niveles (Wood, 2011).

c. La intensificación de las demandas de la planeación de la carrera, que se caracteriza por el incremento de la responsabilidad del empleado para hacerse cargo de su plan de carrera hacia el interior y exterior de su actual organización. En esta dimensión, con los rápidos cambios en el entorno, las personas se ven cada vez más obligadas a ser agentes clave en la generación de valor para sus organizaciones, así como en el incremento de su propia empleabilidad futura. De este modo, un individuo necesita esforzarse cada vez más para mantenerse atractivo para el mercado laboral, lo cual se convierte en una demanda que se caracteriza tanto por la obligación de mantener redes vigentes como por estar abierto a las oportunidades de carrera (Briscoe \& Hall, 2006).

d. La intensificación de las demandas por la adquisición de nuevos aprendizajes, que hace referencia al incremento de la presión para actualizarse y adquirir nuevos conocimientos relacionados con el mundo del trabajo. Con esta demanda, sabiendo que el conocimiento representa un recurso clave en el éxito organizacional y que es la base la competitividad en un mundo complejo, se exige que las personas actualicen sus conocimientos más rápido debido a las innovaciones tecnológicas (Obschonka et al., 2012). 
e. Y, finalmente, la intensificación de las demandas por el desarrollo nuevas competencias, que se caracteriza por un incremento en la necesidad de desarrollar nuevas habilidades para el manejo de equipos, aplicaciones, procesos, metodologías, y para la dirección de equipos de trabajo. Esto debido a que el aprendizaje no solo se orienta al conocimiento, sino que también requiere que las personas desarrollen nuevas habilidades para hacer bien su trabajo; en este sentido, mantener a punto el nivel de competencias se ha vuelto cada vez más demandante (Loon \& Casimir, 2008).

A partir de este modelo, y como resultado de la creciente aceleración en los cambios económicos, sociales y tecnológicos que el mundo del trabajo viene enfrentando, Kubicek et al. (2015) construyeron y validaron el instrumento Intensification of Job Demands Scale (IDS) con el fin de medir la forma en que los individuos perciben las experiencias de intensificación en sus cinco dimensiones conceptuales. De acuerdo con los autores, este instrumento permite capturar directamente la intensificación mediante la valoración del encuestado acerca de la experimentación o no de un aumento en las demandas relacionadas con su tarea, lo que hace que la escala sea una herramienta útil para estudiar los efectos de la intensificación del trabajo sobre la salud y el bienestar de los trabajadores en un contexto organizacional.

Es importante destacar que en la revisión de la literatura especializada no se encuentran otros modelos conceptuales o instrumentos que evalúen el constructo de la intensificación de las demandas, de modo que, como mencionan Riskin et al. (2015), frente a la inexistencia de herramientas para la medición del fenómeno, una opción es utilizar los datos de las encuestas sobre condiciones del trabajo (como la European Working Conditions Survey [EWCS], por ejemplo). De manera relacionada, en la revisión realizada se encontraron algunos instrumentos que permiten evaluar aspectos relacionados con las demandas o condiciones de trabajo, entre los que se encuentran el Job Content Questionnaire (JCQ), de Karaseck et al. (1998), el Effort-Reward Imbalance Questionnaire (ERI), de Siegrist et al. (2004) y el Maslach Bornout Inventory (MBI), de Maslach et al. (1997); sin embargo, a la fecha solo la IDS permite medir el cambio en la intensidad de las demandas.

Ahora bien, teniendo en cuenta que la IDS fue construida originalmente en alemán y en inglés, que se ha utilizado en estudios con población exclusivamente europea (Maunoetal., 2019; Paškvan etal.,2016; Premetal., 2016; Prem et al., 2017), y que hasta el momento no se cuenta con una versión en idioma español que le permita a académicos y profesionales su aplicación en empresas y contextos organizacionales de habla hispana, el objetivo principal del presente estudio fue examinar las evidencias de validez factorial y confiabilidad de una versión traducida al español de la IDS, a partir de una muestra de trabajadores colombianos, con el fin de contribuir al desarrollo de la investigación de esta relevante temática en la región.

\section{Método}

\section{Tipo de estudio}

La presente investigación es de tipo instrumental, según los criterios de Montero y León (2007).

\section{Participantes}

Se contó con la participación de 420 miembros de dos sedes de una organización privada del sector de prestación de servicios de salud en la ciudad de Bogotá, Colombia. Investigaciones previas en el sector (Etzion et al., 1998; Moreno-Jiménez \& Gálvez-Herrer, 2013; Moreno-Jiménez et al., 2009; Sanz-Vergel et al., 2011) lograron establecer efectos negativos de diversos factores asociados a demandas de la tarea y al estrés laboral en personas que trabajan en este tipo de organizaciones, por lo que se encontró adecuado para el objetivo de la investigación utilizar este marco poblacional. Una vez autorizado el estudio por parte del comité de ética de la organización, se realizó un muestreo aleatorio simple para la selección de los participantes sobre un censo total de 1420 personas que tenían contrato permanente, directo y a tiempo completo con la empresa. El número mínimo requerido a partir del muestreo aleatorio para garantizar un intervalo de confianza del $95 \%$ y un nivel de error del $5 \%$ fue de 336 . Finalmente, se obtuvo una participación del $29.6 \%$ del total, en las dos sedes.

Los participantes contaban con edades entre los 18 y los 65 años $(M=35.65 ; D T=8.88)$; 316 eran mujeres y 104 , hombres (75.2 \% y $24.8 \%$ respectivamente); ambos con experiencia laboral variada, entre uno y 43 años $(M=13.74$; $D T=8.2$ ); y también con diversos tiempos en el cargo, oscilando entre nuevos en el cargo y 37 años en el mismo $(M=6.36 ; D T=5.94)$; el $53.1 \%$ refirió tener personal a cargo. Por último, la profesión de los participantes en los cargos administrativos fue en su mayoría de ciencias económicas y administrativas, mientras que para los cargos asistenciales las profesiones eran enfermería, medicina y fisioterapia. En la Tabla 1 se presenta la información del tipo de rol y los tipos de cargo de los participantes. 
Tabla 1.

Tipo de rol y de cargo de los participantes del estudio

\begin{tabular}{lcc}
\hline Tipo de rol & & \\
\hline & Frecuencia & Porcentaje \\
\cline { 2 - 3 } Coordinador & 49 & 11.67 \\
Gerente & 28 & 6.67 \\
Jefe & 65 & 15.47 \\
Profesional & 126 & 30.00 \\
Subgerente & 5 & 1.19 \\
Técnico & 147 & 35.00 \\
\hline
\end{tabular}

Tipo de cargo

\begin{tabular}{|c|c|c|}
\hline & Frecuencia & Porcentaje \\
\hline Administrativo & 284 & 67.63 \\
\hline Asistencial & 93 & 22.14 \\
\hline Directivo & 24 & 5.71 \\
\hline Operativo & 19 & 4.52 \\
\hline
\end{tabular}

Es importante mencionar que se tomó una muestra aleatoria de 200 participantes para llevar a cabo el análisis factorial exploratorio, que el grupo restante de la muestra se utilizó para el análisis confirmatorio, y que se usó la totalidad de la muestra para los análisis de confiabilidad.

\section{Instrumento}

La escala de intensificación de demandas del trabajo [Intensification of Job Demands Scale] (IDS), diseñada por Kubicek et al. (2015), es un instrumento conformado por 19 ítems en escala tipo Likert de cinco puntos -de "totalmente en desacuerdo" (1) a "totalmente de acuerdo" (5)-, agrupados en cinco factores: (a) intensificación del esfuerzo laboral, con cinco ítems y un $\alpha=.91$; (b) intensificación de la planeación y autonomía en el trabajo, con cinco ítems y un $\alpha=.90$; (c) intensificación de la planeación de la carrera, con tres ítems y un $\alpha=.82$; (d) intensificación por adquirir nuevos aprendizajes, con tres ítems y un $\alpha=.90 ; \mathrm{y}$, por último, (e) intensificación por desarrollar nuevas habilidades, con tres ítems y un $\alpha=.87$. Con estos reactivos, el instrumento busca indagar con respecto a la valoración que la persona hace de los cambios en las demandas en su trabajo, más que la disposición del individuo a adaptarse a los mismos.

Cabe destacar que en el estudio original se halló una estructura pentafactorial por medio del análisis factorial confirmatorio, con análisis de modelos rivales y con el método de máxima verosimilitud — modelo de cinco factores con mejor bondad de ajuste- - donde se obtuvieron indicadores apropiados tanto de ajuste comparativo —entre .92 y .96 - como de error cuadrático (RMSEA $=.067-.081$ ), índices de Tucker-Lewis —entre .95 y .93-, análisis de invarianza factorial con muestras alemanas y austriacas — sin cambios significativos en los valores de chi cuadrado para las muestras - análisis de confiabilidad con alfas de Cronbach — valores entre .79 y .94-, análisis de validez convergente con correlaciones a escalas que midieran constructos similares —correlaciones significativas que oscilaban entre .43 y .89 -, validez discriminante con comparaciones entre grupos, y validez incremental con análisis de regresión múltiple.

Para el presente estudio se seleccionó la versión en inglés, y en el Apéndice A se muestra la versión final de la escala en idioma español, la cual se obtuvo gracias a los análisis llevados a cabo en esta investigación.

\section{Procedimiento}

La investigación parte, como lo sugiere Brislin (1970), por un procedimiento de traducción inversa. Para esto, (a) se inició con la traducción al español de los ítems; (b) se procedió a realizar una sesión de grupo con seis profesionales de psicología con énfasis formativo y con experiencia en el campo organizacional y del trabajo, quienes ayudaron en la revisión de los aspectos asociados al lenguaje y extensión de los ítems - todos los ítems fueron aprobados y solo se sugirieron pequeños cambios en la redacción y gramática de los mismos para mejorar el orden sintáctico y el uso de preposiciones - ; y, por último, (c) se tradujo al inglés el instrumento adaptado para comprobar la equivalencia entre las versiones y así completar el proceso de traducción inversa.

Es importante resaltar que, con el fin de mantener el criterio de valoración de la intensidad en el cambio en las demandas del trabajo, más que la disposición personal — como se plantea en la escala original—, se incluyó, en cada uno de los ítems, sintagmas nominales como "cada vez más", con los cuales se buscó denotar la progresión en el cambio de las condiciones del trabajo.

Posteriormente, se obtuvieron los avales de las comisiones éticas tanto de la organización como de la institución académica, y, por último, para la aplicación del instrumento, se utilizó un formato online, que fue enviado por correo electrónico institucional a cada uno de los participantes, junto con la presentación de los objetivos, la participación y las condiciones éticas del estudio, y con el respectivo consentimiento informado y la escala final adaptada.

\section{Análisis de datos}

Una vez terminada la aplicación, se realizó la tabulación de los datos obtenidos y se llevaron a cabo los análisis estadísticos, garantizando inicialmente las condiciones y supuestos para los análisis factoriales - propuestos por Pérez y Medrano (2010)—, como: (a) la eliminación de casos atípicos con la prueba de distancias extremas de Mahalanobis; (b) la normalidad de las variables a través de 
la revisión de la asimetría y la curtosis, cuyos valores debían ser menores $\mathrm{a} \pm 1.5$; (c) la linealidad y las interrelaciones con los análisis de correlaciones, superiores a 0.3 entre pares de ítems de cada subescala; (d) la prueba de Kaiser-MeyerOlkin, con valores esperados superiores a .7; (e) la prueba de esfericidad de Bartlett, con una significancia inferior al .05 ; y, por último, (f) la colinealidad, garantizando que las correlaciones no fueran superiores a .90 .

Posteriormente, se revisó la validez de constructo por medio de un análisis factorial exploratorio, realizado para observar la agrupación libre de reactivos y no vinculada a una estructura ya prediseñada — dado que no existían evidencias factoriales en Colombia-, con un método de extracción de máxima verosimilitud, elegido por tener cinco opciones de respuesta y cumplir con las condiciones de normalidad en todas las variables a factorizar, así como una rotación oblicua, con el método Oblimin, debido a la interrelación de los factores (Lloret-Segura et al., 2014).

A continuación, se realizó un análisis factorial confirmatorio por medio del modelo de ecuaciones estructurales, teniendo en cuenta los siguientes indicadores de bondad de ajuste: $\chi^{2} / \mathrm{gl}<3$, GFI $>.9$, AGFI $>.9$, CFI $>.9$, TLI $>.9$, RMSEA $<.05$. Y, por último, se ejecutó un análisis de confiabilidad con alfas de Cronbach tanto para la escala general como por subescalas, con correlaciones elementoelemento, elemento-total y análisis de confiabilidad si se elimina hipotéticamente cada reactivo. Para la totalidad del análisis se hizo uso del paquete estadístico $\mathrm{R}$-que se usó para el análisis paralelo de Horn—, el software SPSS, versión 24—para los análisis factoriales exploratorios y de confiabilidad—, y el aplicativo AMOS — para el análisis factorial confirmatorio-.

\section{Resultados}

En principio, con respecto a la prueba Kaiser-MeyerOlkin y la prueba de esfericidad de Bartlett para determinar la adecuación de los ítems y de sus interrelaciones para un análisis factorial, los datos mostraron un KMO de $0.801 \mathrm{y}$ una significancia en la esfericidad de 0.000 , lo que da cuenta de la viabilidad de los reactivos y de sus correlaciones para soportar el análisis de factores.

Posteriormente, se llevó a cabo el análisis de comunalidades y extracción para cada reactivo (véase Tabla 2). Como se puede observar, se hallaron valores de extracción superiores a 0.3 para todos los reactivos, a excepción del reactivo 8 , el cual se mantuvo para el resto del análisis factorial con el fin de denotar su viabilidad o decidir finalmente su eliminación.
Tabla 2.

Índices de extracción de cada reactivo

\begin{tabular}{ccc}
\hline & Inicial & Extracción \\
\hline Ítem 1 & .401 & .441 \\
Ítem 2 & .505 & .689 \\
Ítem 3 & .306 & .316 \\
Ítem 4 & .347 & .395 \\
Ítem 5 & .372 & .429 \\
Ítem 6 & .358 & .400 \\
Ítem 7 & .384 & .526 \\
Ítem 8 & .098 & .116 \\
Ítem 9 & .303 & .425 \\
Ítem 10 & .276 & .304 \\
Ítem 11 & .319 & .383 \\
Ítem 12 & .464 & .647 \\
Ítem 13 & .441 & .567 \\
Ítem 14 & .463 & .568 \\
Ítem 15 & .285 & .351 \\
Ítem 16 & .454 & .557 \\
Ítem 17 & .521 & .560 \\
Ítem 18 & .542 & .965 \\
Ítem 19 & .402 & .425 \\
\hline
\end{tabular}

Después, se llevó a cabo el análisis de factores teniendo en cuenta el criterio de Kaiser de autovalores superiores a 1, el análisis paralelo de Horn y la postura teórica original de la escala (Lloret-Segura et al., 2014), donde se hallaron cinco factores que explican el $47.124 \%$ de la varianza total. Los reactivos correspondientes a cada subescala se muestran en la Tabla 3, en la cual se observa que en la distribución de los reactivos se presenta una carga única de cada reactivo a un solo factor, con cargas claramente constituidas (superiores a 0.4 ), a excepción del reactivo 8 , cuya carga es menor al mínimo esperado, a pesar de que esta se presenta en un solo factor.

A continuación, con el fin de verificar la idoneidad del modelo propuesto teóricamente en comparación con modelos alternativos, se realizó un análisis de ecuaciones estructurales, donde se estudiaron los coeficientes del modelo obtenido, así como el análisis de los modelos rivales con el mismo modelo, pero sin el reactivo 8; su similar con cuatro factores; y este último con la eliminación del mismo reactivo (véase Tabla 4). Como se puede observar, el modelo con mejores indicadores de ajuste es el de cinco factores, pero con la eliminación del reactivo 8; modelo que se especifica en la Figura 2.

Ahora, con respecto a la consistencia interna, se halló un alfa de Cronbach estandarizado general de .808 y de .818 sin el reactivo 8 , con correlaciones positivas y superiores a 0.3 entre los reactivos pertenecientes a cada factor, a excepción, nuevamente, del ítem 8 . En la Tabla 5 se muestra el alfa de Cronbach obtenido en cada subescala, 
Tabla 3.

Matriz de patrón obtenida

\begin{tabular}{|c|c|c|c|c|c|c|c|}
\hline & Factor 1 & Factor 2 & Factor 3 & Factor 4 & Factor 5 & Media & Desv. estándar \\
\hline Ítem 2 & 0.883 & & & & & 3.567 & 1.1572 \\
\hline Ítem 1 & 0.661 & & & & & 3.23 & 1.122 \\
\hline Ítem 4 & 0.580 & & & & & 3.676 & 1.1478 \\
\hline Ítem 5 & 0.576 & & & & & 3.836 & 1.0008 \\
\hline Ítem 3 & 0.532 & & & & & 3.598 & 0.9435 \\
\hline Ítem 7 & & 0.686 & & & & 3.819 & 0.9272 \\
\hline Ítem 9 & & 0.661 & & & & 3.145 & 1.0947 \\
\hline Ítem 6 & & 0.490 & & & & 3.712 & 0.9141 \\
\hline Ítem 10 & & 0.482 & & & & 3.493 & 0.9928 \\
\hline Ítem 8 & & 0.263 & & & & 3.386 & 1.1092 \\
\hline Ítem 12 & & & 0.758 & & & 4.240 & 0.6817 \\
\hline Ítem 13 & & & 0.685 & & & 4.110 & 0.7327 \\
\hline Ítem 11 & & & 0.568 & & & 3.950 & 0.9243 \\
\hline Ítem 16 & & & & 0.656 & & 3.886 & 0.8211 \\
\hline Ítem 14 & & & & 0.638 & & 4.055 & 0.8112 \\
\hline Ítem 15 & & & & 0.447 & & 3.448 & 1.0081 \\
\hline Ítem 18 & & & & & 0.995 & 3.826 & 0.8606 \\
\hline Ítem 17 & & & & & 0.463 & 3.893 & 0.7940 \\
\hline Ítem 19 & & & & & 0.444 & 3.733 & 0.9245 \\
\hline
\end{tabular}

Tabla 4.

Coeficientes de modelos obtenido y rivales, en el análisis de ecuaciones estructurales

\begin{tabular}{|c|c|c|c|c|c|c|c|c|}
\hline & $\mathrm{X} 2$ & gl & $\mathrm{X} 2 / \mathrm{gl}$ & GFI & AGFI & TLI & CFI & RMSEA \\
\hline $\begin{array}{l}\text { Cinco factores (propuesta original) con todos } \\
\text { los reactivos }\end{array}$ & 228.943 & 138 & 1.659 & .900 & .862 & .925 & .939 & .055 \\
\hline Cinco factores (propuesta original) sin el reactivo 8 & 182.636 & 121 & 1.509 & .916 & .881 & .947 & .958 & .048 \\
\hline Cuatro factores con todos los reactivos & 243.003 & 136 & 1.787 & .895 & .853 & .910 & .929 & .060 \\
\hline Cuatro factores sin el reactivo 8 & 196.575 & 119 & 1.652 & .911 & .872 & .932 & .947 & .055 \\
\hline
\end{tabular}

Nota. $\mathrm{X}^{2}=$ chi cuadrado; gl = grados de libertad; GFI = índice de bondad de ajuste; AGFI = índice de bondad de ajuste estandarizado; TLI = índice Tucker-Lewis; CFI = índice de ajuste comparativo; RMSEA = error cuadrático medio de aproximación de la raíz.

así como las correlaciones entre cada reactivo y el total, y el alfa si se elimina hipotéticamente el reactivo. Con estos resultados se evidencian correlaciones positivas superiores a 0.4 entre cada reactivo y el total, además de alfas que se afectan ante la eliminación de los reactivos, a excepción del reactivo 8 , lo que rectifica la necesidad de eliminar este ítem. Incluso, desde la perspectiva teórica se puede afirmar que al eliminar este ítem no se pierde la capacidad del instrumento para medir la intensificación del nivel de autonomía en la realización de las tareas, ya que los otros cuatro reactivos del factor permiten capturar la experiencia del individuo en la dimensión evaluada.

Finalmente, y con el propósito de evaluar la consistencia interna de cada uno de los componentes de la escala, se realizó el cálculo de la fiabilidad compuesta para cada factor (IFC) (Fornell \& Larcker, 1981), en el cual se tienen en cuenta las interrelaciones entre los ítems, para una mayor consistencia y una mejor medida de la fiabilidad. Como se evidencia en la Tabla 5, los valores del IFC de la escala superan el límite de 0.70 recomendado en la literatura (Hair et al., 1999).

\section{Discusión}

Estudios recientes han demostrado que la intensificación de las demandas y exigencias en el mundo del trabajo cada vez son mayores, razón por la cual se hace necesario gestionar el efecto de estas sobre trabajadores y organizaciones. Por ello, es importante contar con instrumentos de evaluación y medición óptimos, válidos y confiables para su adecuado diagnóstico y posterior intervención en los contextos organizacionales.

Teniendo esta problemática en cuenta, el presente trabajo tuvo el objetivo de traducir y validar la Intensification 


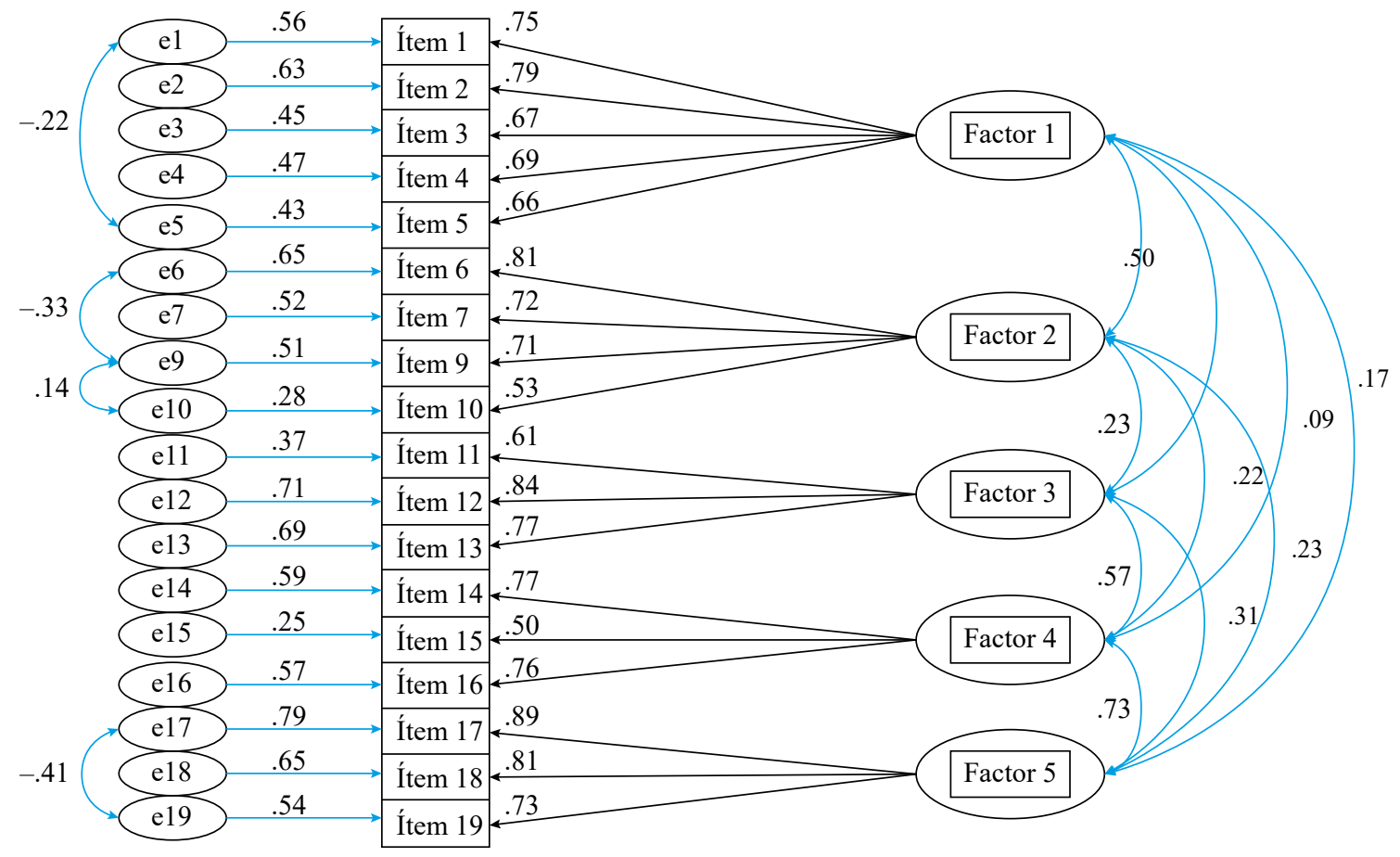

Figura 2. Modelo pentafactorial sin el octavo reactivo.

Tabla 5.

Estadísticos de confiabilidad para cada factor

\begin{tabular}{|c|c|c|c|c|c|}
\hline & $\begin{array}{l}\text { Alfa del } \\
\text { factor }\end{array}$ & Reactivos & $\begin{array}{c}\text { Correlación } \\
\text { ítem-total }\end{array}$ & $\begin{array}{l}\text { Alfa si el elemento } \\
\text { se elimina }\end{array}$ & $\begin{array}{l}\text { Confiabilidad } \\
\text { compuesta IFC }\end{array}$ \\
\hline \multirow{5}{*}{$\begin{array}{l}\text { Intensificación de demandas de esfuerzo } \\
\text { en el trabajo }\end{array}$} & \multirow{5}{*}{.787} & Ítem 1 & .578 & .744 & \multirow{5}{*}{.832} \\
\hline & & Ítem 2 & .678 & .708 & \\
\hline & & Ítem 3 & .503 & .768 & \\
\hline & & Ítem 4 & .547 & .755 & \\
\hline & & Ítem 5 & .526 & .76 & \\
\hline \multirow{5}{*}{$\begin{array}{l}\text { Intensificación de demandas de planeación } \\
\text { y autonomía en el trabajo }\end{array}$} & \multirow{5}{*}{.654} & Ítem 6 & .438 & .57 & \multirow{5}{*}{.797} \\
\hline & & Ítem 7 & .557 & .514 & \\
\hline & & Ítem 8 & .149 & .713 & \\
\hline & & Ítem 9 & .485 & .54 & \\
\hline & & Ítem 10 & .407 & .582 & \\
\hline \multirow{3}{*}{$\begin{array}{l}\text { Intensificación de demandas para la } \\
\text { planeación de carrera }\end{array}$} & \multirow{3}{*}{.754} & Ítem 11 & .516 & .743 & \multirow{3}{*}{.748} \\
\hline & & Ítem 12 & .613 & .611 & \\
\hline & & Ítem 13 & .594 & .62 & \\
\hline \multirow{3}{*}{$\begin{array}{l}\text { Intensificación de demandas para adquirir } \\
\text { nuevos aprendizajes }\end{array}$} & \multirow{3}{*}{.702} & Ítem 14 & .533 & .479 & \multirow{3}{*}{.74} \\
\hline & & Ítem 15 & .559 & .433 & \\
\hline & & Ítem 16 & .536 & .473 & \\
\hline \multirow{3}{*}{$\begin{array}{l}\text { Intensificación de demandas por } \\
\text { desarrollar nuevas competencias }\end{array}$} & \multirow{3}{*}{.797} & Ítem 17 & .628 & .731 & \multirow{3}{*}{.749} \\
\hline & & Ítem 18 & .704 & .645 & \\
\hline & & Ítem 19 & .587 & .779 & \\
\hline
\end{tabular}


of Job Demands Scale (IDS) [escala de intensificación de demandas del trabajo], diseñada por Kubicek et al. (2015), en el contexto colombiano. Los resultados del estudio muestran que la escala, en su versión en español, presenta un modelo de cinco factores que logran explicar el $47.124 \%$ de la varianza total, con una agrupación de reactivos similar a la propuesta teórica original, y con altos coeficientes de consistencia y confiabilidad, aunque con la recomendación de que para esta versión del instrumento se elimine el ítem 8.

Estos hallazgos son similares a los encontrados en el estudio original la escala (Kubicek et al., 2015), donde también se encuentra una estructura pentafactorial, y con la misma distribución de reactivos, índices de bondad de ajuste e indicadores de confiabilidad similares, lo que reafirma la equivalencia del constructo y da muestras adicionales para referir que la escala es válida y confiable para evaluar la intensificación de las demandas en la tarea y el trabajo en contextos empresariales de habla hispana. Cabe resaltar que, debido a la actualidad del constructo y la novedad de la escala, la literatura para comparar los hallazgos es bastante limitada.

Por otra parte, también es importante señalar algunas limitaciones del presente estudio, como que, si bien el tamaño muestral fue suficiente para soportar los análisis estadísticos, el mismo no es representativo de la unidad poblacional, ya que la muestra pertenece a una misma organización y está compuesta principalmente por mujeres $(75.2 \%)$; razón por la cual se recomienda la replicación de esta investigación con otras muestras de mayor tamaño y diversidad poblacional. De igual forma, la muestra fue obtenida de solo un sector económico, por lo que se sugiere hacer replicaciones con muestras de otros sectores empresariales. Y, por último, la comparación de los resultados resulta difícil debido a que, a la fecha, no se han realizado otros estudios en la región para analizar sus propiedades psicométricas; por lo cual se sugiere la réplica del estudio en otros contextos y países como línea futura de investigación.

Como conclusión, puede referirse que la versión en español de la Intensification of Job Demands Scale (IDS) es una escala válida, consistente y confiable para evaluar directamente el constructo, de modo que se recomienda su uso para medir las nuevas demandas que surgen en el mundo del trabajo debidas a la aceleración social, y de este modo evaluar la percepción de cambio en las condiciones laborales de un contexto particular, así como los efectos asociados a la aceleración de los ritmos de gestión sobre la salud y el bienestar de los trabajadores en un contexto organizacional.

\section{Referencias}

Bakker, A. B., \& Demerouti, E. (2007). The job demands -resources model: State of the art. Journal of Managerial Psychology, 22(3), 309-328. https://doi. org/10.1108/02683940710733115

Bakker, A. B., \& Demerouti, E. (2014). Job DemandsResourses Theory. En P. Y. Chen \& C. L. Cooper (Eds.), Work and Wellbeing: Wellbeing: A Complete Reference Guide (vol. III). John Wiley \& Sons, Inc. https://doi. org/10.1002/9781118539415.wbwell019

Bakker, A. B., Demerouti, E., \& Schaufeli, W. (2003). Dual processes at work in a call centre: An application of the job demands-resources model. European Journal of work and organizational psychology, 12(4), 393-417. https://doi. org/10.1080/13594320344000165

Bakker, A. B., Hakanen, J. J., Demerouti, E., \& Xanthopoulou, D. (2007). Job resources boost work engagement, particularly when job demands are high. Journal of Educational Psychology, 99(2), 274. https://doi. org/10.1037/0022-0663.99.2.274

Bathini, D. R., \& Kandathil, G. M. (2019). An Orchestrated Negotiated Exchange: Trading Home-Based Telework for Intensified Work. Journal of Business Ethics, 154(2), 411423. https://doi.org/10.1007/s10551-017-3449-y

Briscoe, J. P., \& Hall, D. T. (2006). The interplay of boundaryless and protean careers: Combinations and implications. Journal of Vocational Behavior, 69(1), 4-18. https://doi. org/10.1016/j.jvb.2005.09.002

Brislin, R. W. (1970). Back-Translation for Cross-Cultural Research. Journal of Cross-Cultural Psychology, 1(3), 185216. https://doi.org/10.1177/135910457000100301

Etzion, D., Eden, D., \& Lapidot, Y. (1998). Relief from job stressors and burnout: Reserve service as a respite. Journal of Applied Psychology, 83(4), 577-585. https://doi. org/10.1037/0021-9010.83.4.577

Flecker, J., Fibich, T., \& Kraemer, K. (2017). Socio-Economic Changes and the Reorganization of Work. In Job Demands in a Changing World of Work (pp. 7-24). Springer. https:// doi.org/10.1007/978-3-319-54678-0_2

Fornell, C., \& Larcker, D. F. (1981). Evaluating structural equation models with unobservable variables and measurement error. Journal of Marketing Research, 18(1), 39-50. https:// doi.org/10.2307/3151312

Franke, F. (2015). Is work intensification extra stress? Journal of Personnel Psychology, 14(1), 17-27. https://doi. org/10.1027/1866-5888/a000120

Granter, E., Wankhade, P., McCann, L., Hassard, J., \& Hyde, P. (2019). Multiple Dimensions of Work Intensity: Ambulance Work as Edgework. Work, Employment and Society, 33(2), 280-297. https://doi.org/10.1177/0950017018759207

Hair, J. F., Anderson, R. E., Tatham, R. L., \& Black, W. C. (1999). Análisis multivariante (vol. 491). Prentice Hall. 
Hakanen, J. J., Bakker, A. B., \& Schaufeli, W. B. (2006). Burnout and work engagement among teachers. Journal of School Psychology, 43(6), 495-513. https://doi.org/10.1016/j. jsp.2005.11.001

Karasek, R., Brisson, C., Kawakami, N., Houtman, I., Bongers, P., \& Amick, B. (1998). The Job Content Questionnaire (JCQ): an instrument for internationally comparative assessments of psychosocial job characteristics. Journal of Occupational Health Psychology, 3(4), 322-355. https:// doi.org/10.1037/1076-8998.3.4.322

Kelliher, C., \& Anderson, D. (2008). For better or for worse? An analysis of how flexible working practices influence employees' perceptions of job quality. The International Journal of Human Resource Management, 19(3), 419-431. https://doi.org/10.1080/09585190801895502

Krause, N., Scherzer, T., \& Rugulies, R. (2005). Physical workload, work intensification, and prevalence of pain in low wage workers: Results from a participatory research project with hotel room cleaners in Las Vegas. American Journal of Industrial Medicine, 48(5), 326-337. https://doi. org/10.1002/ajim.20221

Kubicek, B., Korunka, C., \& Ulferts, H. (2013). Acceleration in the care of older adults: new demands as predictors of employee burnout and engagement. Journal of Advanced Nursing 69(7), 1525-1538. https://doi.org/10.1111/ jan.12011

Kubicek, B., Paškvan, M., \& Korunka, C. (2015). Development and validation of an instrument for assessing job demands arising from accelerated change: The intensification of job demands scale (IDS). European Journal of Work and Organizational Psychology, 24(6), 898-913. https://doi.org/ 10.1080/1359432X.2014.979160

Lesener, T., Gusy, B., \& Wolter, C. (2019). The job demandsresources model: A meta-analytic review of longitudinal studies. Work and Stress, 33(1), 76-103. https://doi.org/10 $.1080 / 02678373.2018 .1529065$

Lloret-Segura, S., Ferreres-Traver, A., Hernández-Baeza, A., \& Tomás-Marco, I. (2014). El análisis factorial exploratorio de los ítems: una guía práctica, revisada y actualizada. Anales de Psicología, 30(3), 1151-1169. https://doi.org/10.6018/ analesps.30.3.199361

Loon, M., \& Casimir, G. (2008). Job-demand for learning and job-related learning: the moderating effect of need for achievement. Journal of Managerial Psychology, 23(1), 89102. https://doi.org/10.1108/02683940810849684

Maslach, C., Jackson, S. E., \& Leiter, M. P. (1997). Maslach Burnout Inventory: Third edition. En C. P. Zalaquett \& R. J. Wood (Eds.), Evaluating stress: A book of resources (pp. 191-218). Scarecrow Education.

Mauno, S., Kubicek, B., Minkkinen, J., \& Korunka, C. (2019). Antecedents of intensified job demands: evidence from Austria. Employee Relations, 41(4), 694-707. https://doi. org/10.1108/ER-04-2018-0094
Montero, I., \& León, O. (2007). Guía para nombrar los estudios de investigación en Psicología. International Journal of Clinical and Health Psychology, 7(3), 847-862. http:// www.aepc.es/ijchp/GNEIP07_es.pdf

Moreno-Jiménez, B., \& Gálvez-Herrer, M. (2013). El efecto del distanciamiento psicológico del trabajo en el bienestar y la satisfacción con la vida: Un estudio longitudinal [The effect of psychological detachment from work on well-being and life satisfaction: A longitudinal study]. Journal of Work and Organizational Psychology, 29(3), 145-151. https://doi. org/10.5093/tr2013a20

Moreno-Jiménez, B., Mayo, M., Sanz-Vergel, A. I., Geurts, S., Rodríguez-Muñoz, A., \& Garrosa, E. (2009). Effects of Work-Family Conflict on Employees' Well-Being: The Moderating Role of Recovery Strategies. Journal of Occupational Health Psychology, 14(4), 427-440. https:// doi.org/10.1037/a0016739

Neirotti, P. (2018). Work intensification and employee involvement in lean production: new light on a classic dilemma. International Journal of Human Resource Management, 1-26. https://doi.org/10.1080/09585192.2018.1424016

Obschonka, M., Silbereisen, R. K., \& Wasilewski, J. (2012). Constellations of new demands concerning careers and jobs: Results from a two-country study on social and economic change. Journal of Vocational Behavior, 80(1), 211223. https://doi.org/10.1016/j.jvb.2011.08.002

Paškvan, M., \& Kubicek, B. (2017). The Intensification of Work. En C. Korunka \& B. Kubicek (Eds.), Job demands in a changing world of work: Impact on workers' health and performance and implications for research and practice (pp. 25-43). Springer International Publishing. https://doi. org/10.1007/978-3-319-54678-0

Paškvan, M., Kubicek, B., Prem, R., \& Korunka, C. (2016). Cognitive appraisal of work intensification. International Journal of Stress Management, 23(2), 124. https://doi. org/10.1037/a0039689

Pérez, E. R., \& Medrano, L. A. (2010). Análisis factorial exploratorio: bases conceptuales y metodológicas. Revista Argentina de Ciencias del Comportamiento (RACC), 2(1), 58-66. https://doi.org/10.32348/185

Prem, R., Kubicek, B., Diestel, S., \& Korunka, C. (2016). Regulatory job stressors and their within-person relationships with ego depletion: The roles of state anxiety, self-control effort, and job autonomy. Journal of Vocational Behavior, 92, 22-32. https://doi.org/10.1016/j. jvb.2015.11.004

Prem, R., Ohly, S., Kubicek, B., \& Korunka, C. (2017). Thriving on challenge stressors? Exploring time pressure and learning demands as antecedents of thriving at work. Journal of Organizational Behavior, 38(1), 108-123. https://doi. org/10.1002/job.2115

Riskin, A., Erez, A., Foulk, T. A., Kugelman, A., Gover, A., Shoris, I., ... Bamberger, P. A. (2015). The impact of 
rudeness on medical team performance: A randomized trial. Pediatrics, 136(3), 487-495. https://doi.org/10.1542/ peds.2016-2305

Rosa, H. (2003). Social acceleration: ethical and political consequences of a desynchronized high-speed society. Constellations, 10(1), 3-33. https://doi.org/10.1111/1467 $-8675.00309$

Rosa, H. (2013). Social acceleration: A new theory of modernity. Columbia University Press. https://doi.org/10.7312/ rosa14834

Sanz-Vergel, A. I., Demerouti, E., Bakker, A. B., \& MorenoJiménez, B. (2011). Daily detachment from work and home:
The moderating effect of role salience. Human Relations, 64(6), 775-799. https://doi.org/10.1177/0018726710393368

Siegrist, J., Starke, D., Chandola, T., Godin, I., Marmot, M., Niedhammer, I., \& Peter, R. (2004). The measurement of effort-reward imbalance at work: European comparisons. Social Science and Medicine, 58(8), 1483-1499. https://doi. org/10.1016/S0277-9536(03)00351-4

Wood, L. A. (2011). The Changing Nature of Jobs: A Metaanalysis Examining Changes in Job Characteristics Over Time. University of Georgia, Athens. https://getd.libs.uga. edu/pdfs/wood_lauren_a_201105_ms.pdf 


\section{Versión en español de la escala de intensificación de demandas del trabajo (Intensification of Job Demands Scale - IDS)}

Intensificación de demandas del esfuerzo laboral

En esta organización ...

1. Es cada vez más difícil para mí tener suficiente tiempo para las tareas de trabajo.

2. Me es cada vez más difícil tomar tiempo para los descansos.

3. Cada vez es menor el tiempo entre las fases de mayor intensidad en el trabajo.

4. Cada vez es más frecuente hacer dos o tres cosas a la vez (p. ej.: almorzar, escribir correos y hablar por teléfono).

5. Cada vez hay más trabajo y menos personas para realizarlo.

Intensificación de demandas de la planeación y de autonomía en el trabajo

En esta organización...

6. Cada vez más requiero planificar mis actividades de trabajo de manera autónoma.

7. Cada vez más defino por mí mismo la forma en que hago el trabajo.

9. Cada día más tomo las decisiones de mi puesto sin estar consultando a los jefes.

10. Cada vez más debo comprobar por mí mismo si he alcanzado los objetivos del trabajo.

Intensificación de demandas de la planeación de la carrera

En esta organización ...

11. Cada vez tengo mayor exigencia para mantenerme atractivo para el mercado laboral (p. ej.: educación avanzada, obtener certificaciones, pertenecer a redes).

12. Mi propio desarrollo profesional requiere cada vez más estar al tanto de nuevas oportunidades de actualización.

13. Cada vez más debo planear mi carrera profesional por mí mismo.

Intensificación de demandas por adquirir nuevos aprendizajes

En esta organización ...

14. Debo adquirir nuevos conocimientos para mi trabajo más a menudo.

15. Recibo cada vez más nuevos conocimientos para realizar las tareas de mi trabajo.

16. Debo actualizar mis niveles de conocimiento con mayor frecuencia.

Intensificación de demandas por desarrollar nuevas habilidades

En esta organización...

17. Cada vez más existen nuevos temas en el trabajo con los que debo familiarizarme.

18. Cada vez más debo aprender nuevos procesos y flujos de trabajo.

19. Debo usar nuevas herramientas de trabajo más a menudo (p. ej.: programas, equipos, aplicativos, etc.). 